\title{
Follow up pattern for post oesophagectomy patients at a single centre: association with peri-operative variables
}

S.W.O. Ogendo, MBChB, MMed (Surg), Associate Professor, Department of Surgery, College of Health Sciences, University of Nairobi, P.O. Box 19676-00202, Nairobi, Kenya

\section{Abstract}

BACKGROUND: Though the operative mortality for resection of oesophageal malignancy has fallen significantly over time, the overall postoperative survival has remained fairly constant irrespective of surgical techniques, preoperative disease spread being the main independent prognostic indicator of survival.

AIM: This is a five year prospective review, from 2001 to 2006, of post oesophagectomy patients, evaluating follow up and associated variables.

MATERIALS AND METHODS: One hundred and seven patients were included into the study. Peri-operative and post discharge data were recorded. Data analysis reviewed the follow up pattern and the relationship to selected variables.

RESULTS: The one, three and five year follow up rates were $30 \%, 5 \%$ and $3 \%$ respectively. Although a trend of shorter follow up was noted for the presence of preoperative overweight, $\left(<25 \mathrm{Kg} / \mathrm{m}^{2}\right)$, postoperative anastomotic leak and signs of nodal spread of disease, only low preoperative serum protein and albumin levels were significantly associated with a shortened follow up. Seven patients in this series developed recurrent signs of malignant disease.

CONCLUSION: The trends of association of peri-operative variables and postoperative follow up were similar to the international literature on the postoperative survival pattern. Even though this study failed to achieve statistical significance associating disease spread with shortened follow up, improved preoperative staging remains an important goal for better post operative follow up in this region.

\section{Introduction}

Data on patient survival post oesophagectomy from different centres varies widely (5-year survival rates of $7 \%$ to $86 \%$ with an average of $21 \%)(1-4)$.

This wide variation results from the inclusion of both early and late stages of disease in the different reports and differing histology. Illustrating this, King et al. have demonstrated that stage I tumour patients had a survival advantage of up to six times over stage III tumour patients (5).

There have been attempts over the years to reduce operative mortality of oesophageal surgery and to identify factors associated with improved postoperative survival. Though the operative mortality for oesophageal resection has fallen over time, the overall postoperative survival has remained fairly constant irrespective of surgical techniques, the average survival time being about 6 months once metastasis is present $(6,7)$. A review of the situation in a 
single institution in Japan reports improved 5 year survival from $31 \%$ in 1988 to $41 \%$ in 1994 as a result of addressing some of these variables relating to improved postoperative survival (8).

Post operative survival and follow up are not necessarily the same thing but may have similar trends. Valid survival data in our region is hampered by poor communication with patients post discharge. This study is a five year prospective review of the follow up of post oesophagectomy patients at the Kenyatta National Hospital, (KNH), Nairobi, and analyses the effect of selected peri-operative variables on the follow up pattern.

\section{Materials and methods}

This was a prospective study covering the period from February 2001 to January 2006.

All post oesophagectomy patients discharged from the cardiothoracic unit at the $\mathrm{KNH}$ were included. The oesophagectomies were performed using the McEven, Ivor Lewis or trans hiatal resection procedures and the selection of the procedure was at the discretion of the surgeon. For purposes of this study all resections are considered to be palliative therapies and no regional nodal dissection procedures were attempted.

Preoperative parameters evaluated included weight and height to calculate the body mass index (BMI), full haemogram, urea and electrolytes and liver function tests. Additional data included patient demographics, distance of their homes from the Kenyatta National Hospital, the duration of dysphagia and endoscopic and histological information.

At surgery, information collected included the visual extent of enlarged paraoesophageal and coeliac nodes. Where significantly enlarged, samples of the nodes were taken with the oesophagectomy specimen for histological examination.

During post discharge clinic follow up, any complications that may have occurred and patient weight on the day of visit were recorded. For postoperative weight change a difference of half a unit or more in the BMI compared to the previous recording was arbitrarily considered to be significant change.
Wherea postoperativehistologicalspecimen report on the excised oesophagus was present, data on tumour spread through the oesophageal wall and also involvement of the surrounding nodes plus margins was documented. The post resection histology reports of the degree of tumour differentiation, was used for purposes of final analysis, if different to the preoperative endoscopic result.

Data analysis included the determination of the follow up pattern and the variables having an influence on the follow-up pattern. The study end point was 31st January 2006. A patient was considered to have reached the end point when the he/she failed to attend clinic follow up for a period of more than three months from the last visit.

Statistical analysis was performed using statistical Package for Social Sciences, (SPSS), version 11.5. Analysis for follow-up data was determined using the Kaplan Mayer method. For the analysis of relationship of follow up and the different variables, the Log Rank test for significance between curves was adopted for categorical data while linear regression model was used for numerical data. Comparison of means was assessed using the student t-test.

Significance was considered achieved with a $p$ value of less than 0.05 for the above calculations.

\section{Results}

Within the study period the total number of oesophagectomies performed were 129 cases, while those fulfilling the inclusion criteria for the study were 107 patients. The average age for this sample was $57.9 \pm 13.9$ years with a male: female ratio of 1.8:1.

Final post discharge follow up pattern for all patients is represented in figure 1 . Half the patients had dropped out to follow up at seven and a half months. The respective follow up rates for the one, three and five years of follow up were $30 \%, 5 \%$ and three percent.

The occurrence of a postoperative leak ( $p=$ $0.0786)$, a greater number of surrounding enlarged nodes located near the tumour, $(p=0.414)$, the invasion of tumour through the oesophageal serosal wall, $(p=0.0622)$ all seemed to have a 
comparatively shorter follow up compared to patients without leaks or lesser tumour invasion. Despite this apparent picture none of the results were statistically significant; however serosal invasion and post operative anastomotic leak almost attained significance. Also showing an apparent shorter follow up was the resection of tumour using the transhiatal approach over transthoracic, this too howeverwas not statistically significant $(p=0.2505)$ (Figures $2,3,4,5)$.
The degree of histological differentiation showed no significant relationship with the follow-up pattern for squamous cell carcinoma ( $\mathrm{p}=0.1597)$.

Those patients who were overweight preoperatively, $\left(\mathrm{BMI}>25 \mathrm{~kg} / \mathrm{m}^{2}\right)$, displayed a shorter follow up compared to normal, $(20 \mathrm{~kg} /$ $\left.\mathrm{m}^{2}-25 \mathrm{~kg} / \mathrm{m}^{2}\right)$, or underweight, $\left(<20 \mathrm{~kg} / \mathrm{m}^{2}\right)$, patients $(p=0.0816)$, (Figure 7).

Figure 1: Follow up pattern: all oesophagectomies

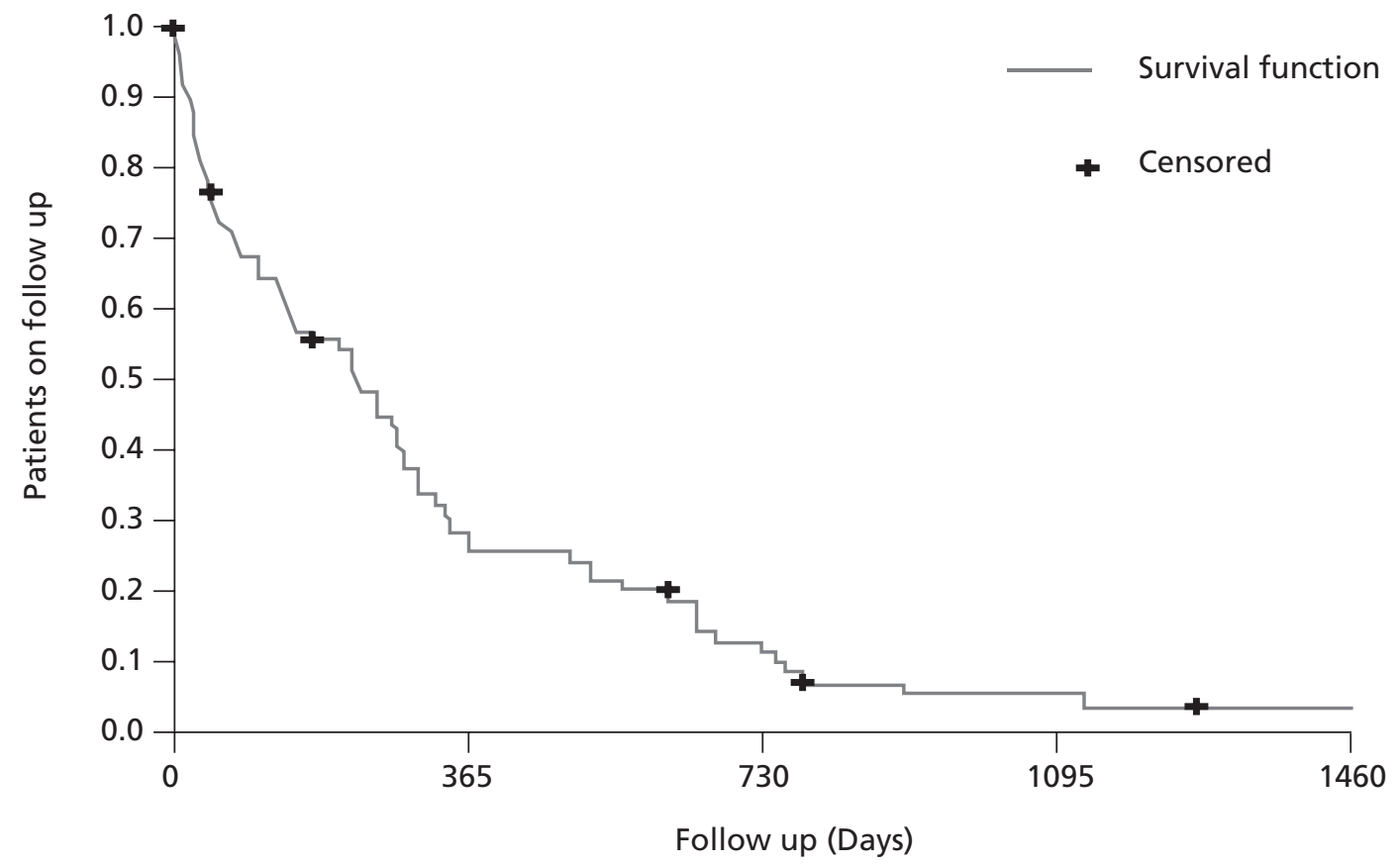

Figure 2: Postoperative leak occurrence and follow up

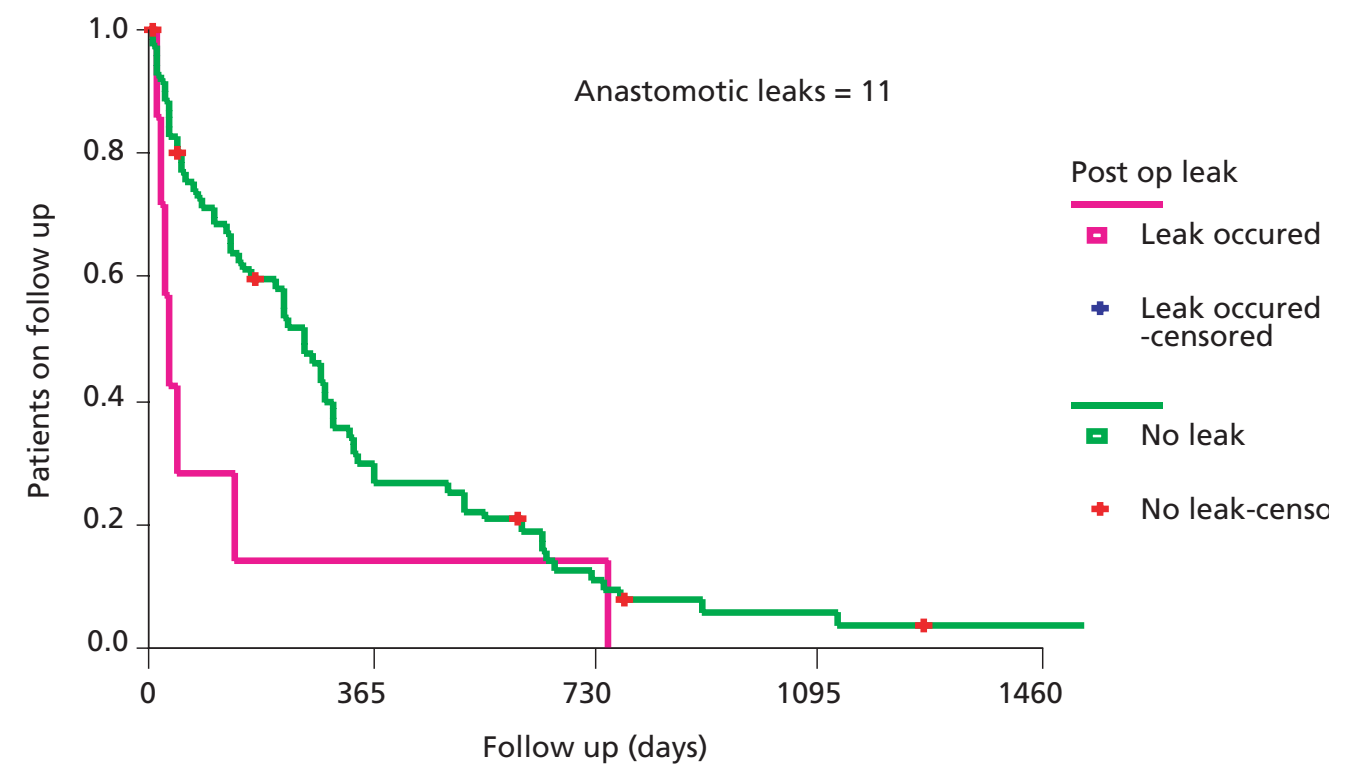


Figure 3: Number of enlarged surrounding lymph nodes and postoperative follow up

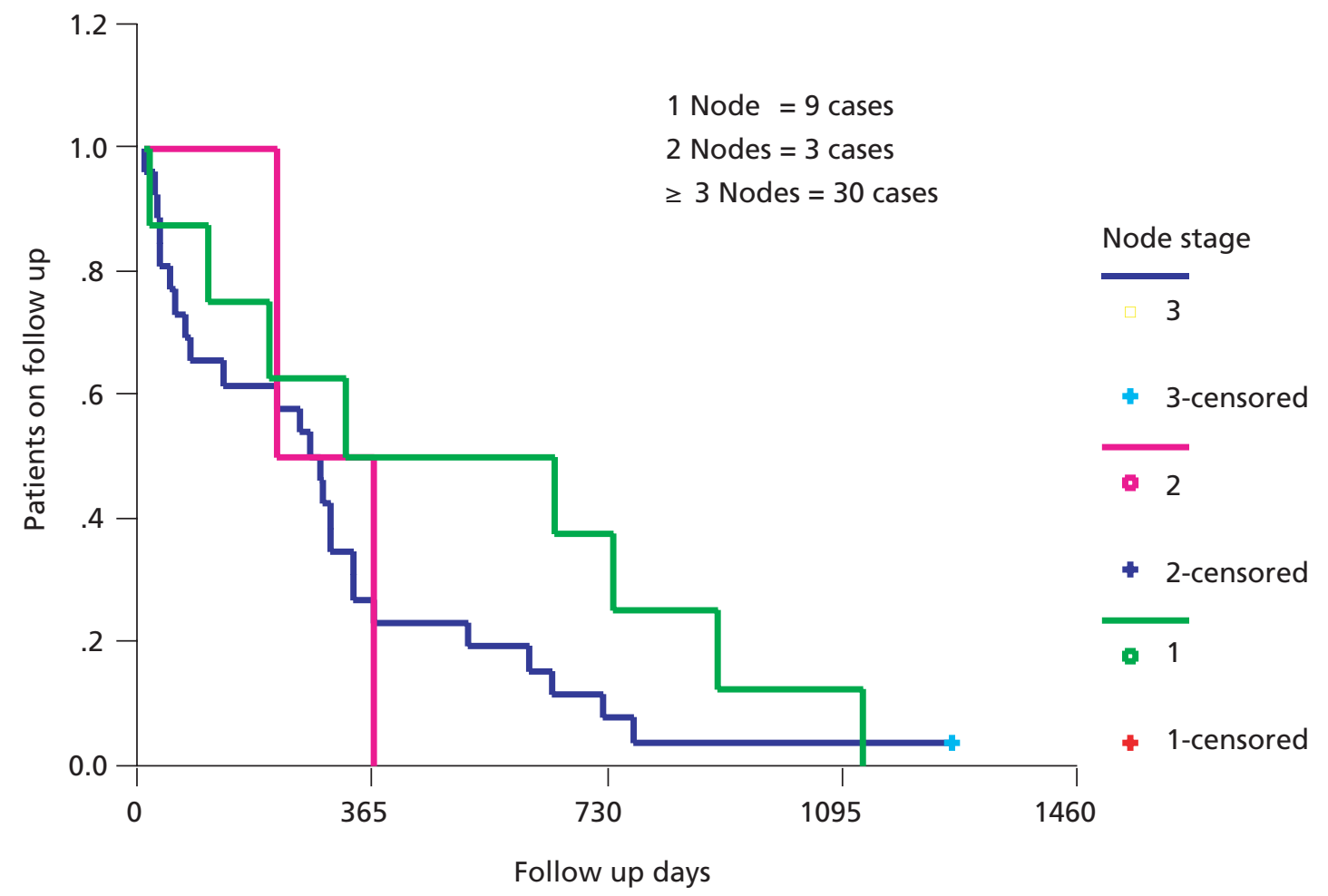

Figure 4: Presence of serosal invasion and postoperative follow up

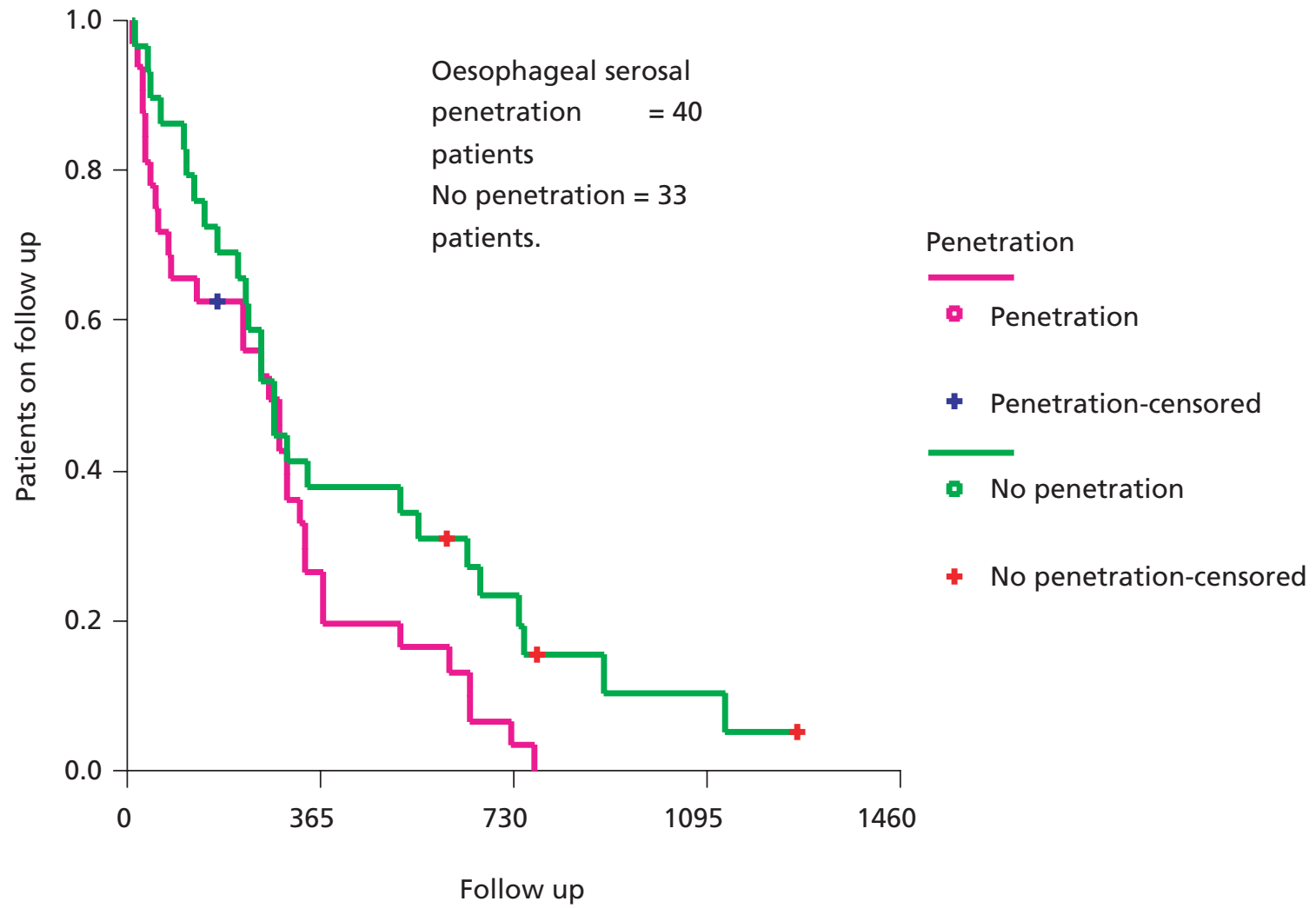


Figure 5: Surgical approach and postoperative follow up

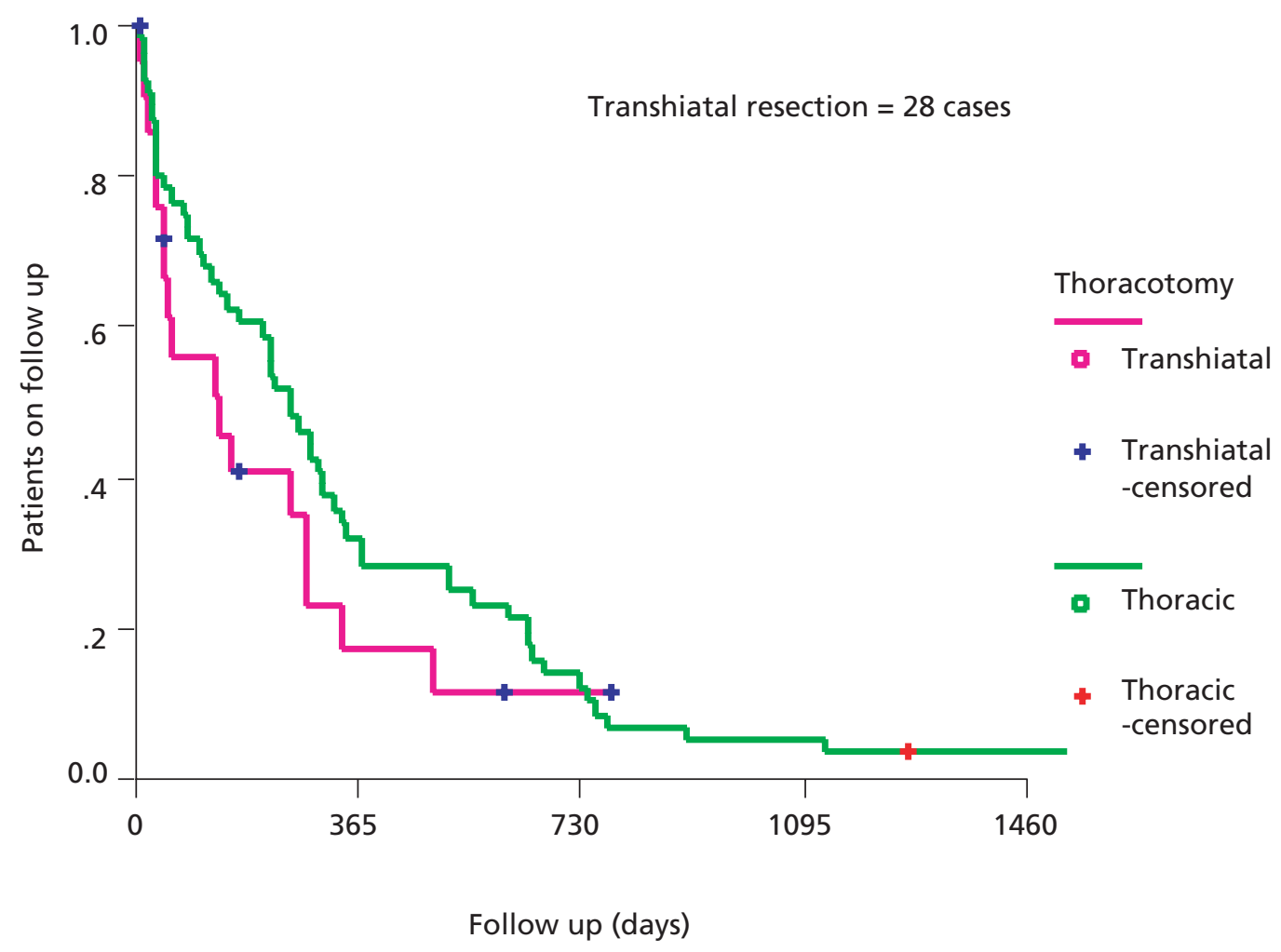

Figure 6: Degree of cellular differentiation and follow up

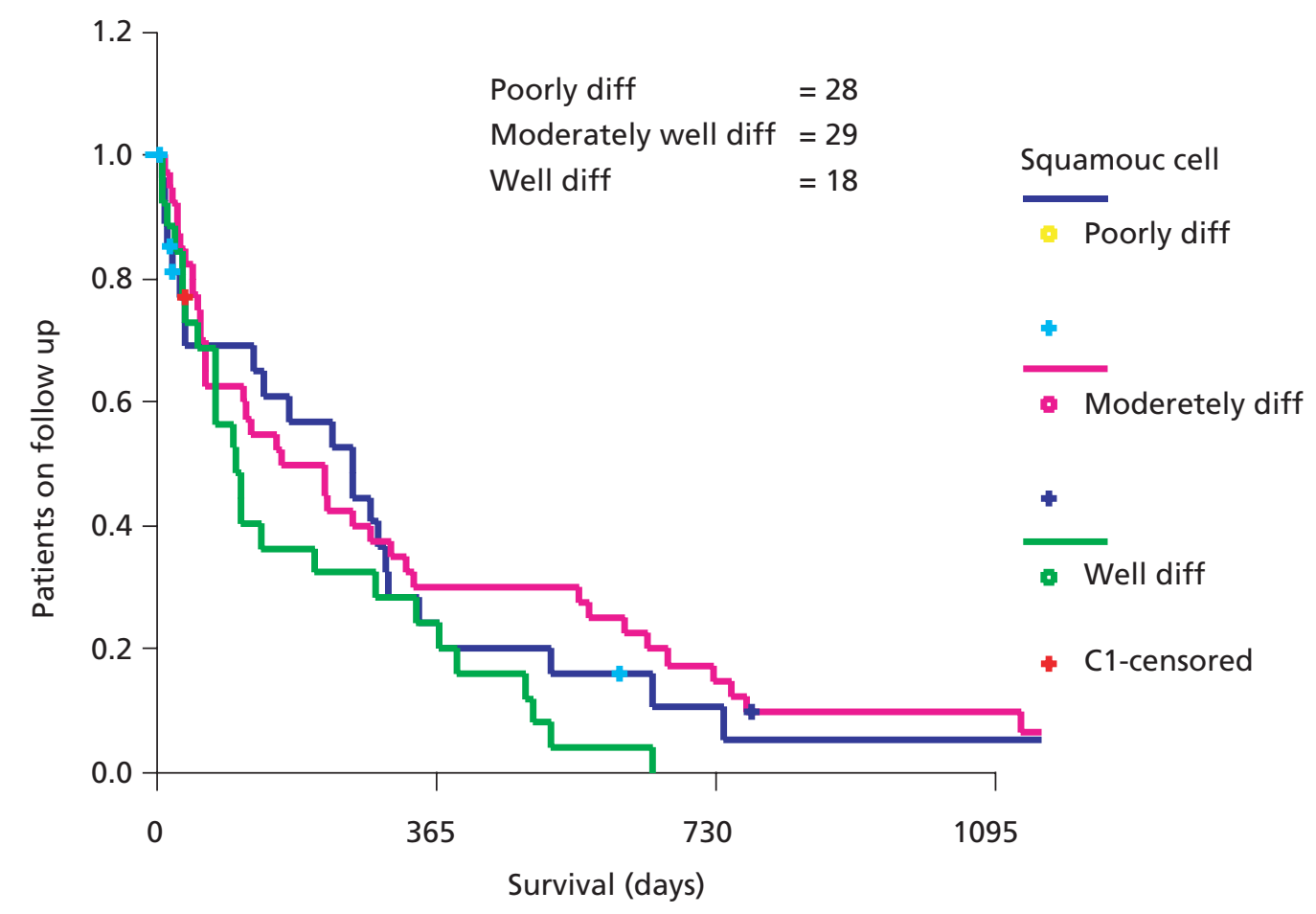


The variables of patient age, tumour level on endoscopy, preoperative serum haemoglobin, billirubin, protein, and albumin levels; as well as the duration of symptoms of dysphagia and the distance of patients residence from the Kenyatta National Hospital, (as the crow flies), were analysed against follow up pattern. Only low serum albumin and protein levels were significantly related to follow up with serum creatinine falling just outside the level of significance (Table 1).

\section{Figure 7: Pre-operative BMI and postoperative follow up}

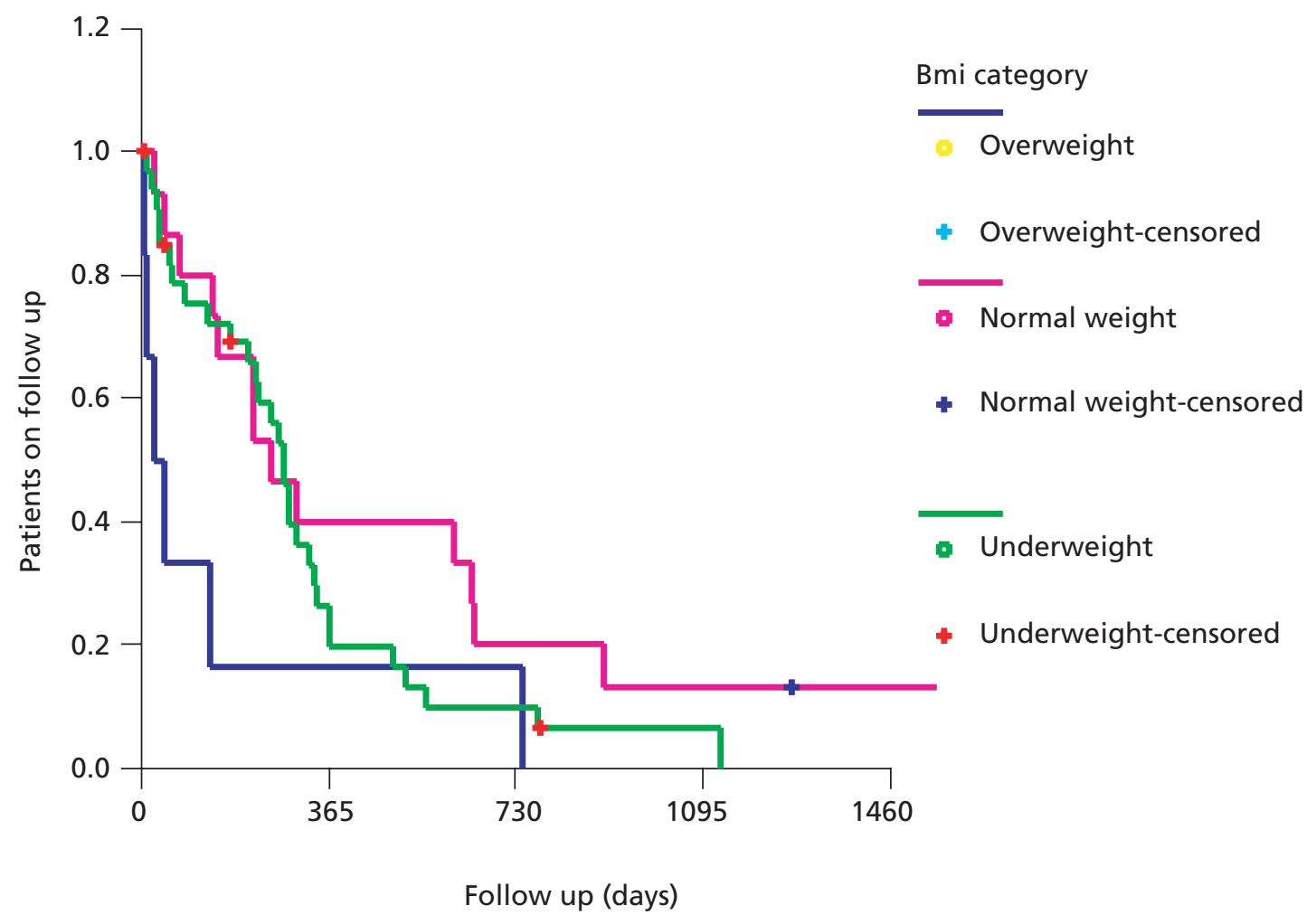

Table 1: Comparison of follow up and numerical variables

coefficients (a)

\begin{tabular}{|c|c|c|c|c|c|c|}
\hline \multicolumn{2}{|c|}{ Model } & \multicolumn{2}{|c|}{ Unstandardised coefficients } & \multirow{2}{*}{$\begin{array}{c}\text { Standardized } \\
\text { coefficients } \\
\text { Beta }\end{array}$} & \multirow{2}{*}{$\mathrm{t}$} & \multirow{2}{*}{ Sig. } \\
\hline & & $B$ & Std. Error & & & \\
\hline \multirow[t]{11}{*}{1} & (Constant) & 4985.521 & 4153.910 & & 1.200 & .248 \\
\hline & Age & -21.078 & 26.240 & -.176 & -.803 & .434 \\
\hline & Distance to $\mathrm{KNH}$ & -3.804 & 3.990 & -.226 & -.953 & .355 \\
\hline & Serum haemoglobin & -373.937 & 269.909 & -.349 & -1.385 & .185 \\
\hline & Serum BUN & 42.655 & 151.253 & .063 & .282 & .782 \\
\hline & Serum creatinine & 29.485 & 14.269 & .456 & 2.066 & .055 \\
\hline & Serum protein & -116.254 & 40.324 & -.703 & -2.883 & .011 \\
\hline & Serum albumin & 197.056 & 63.942 & .749 & 3.082 & .007 \\
\hline & Total serum billirubin & 44.735 & 53.814 & .181 & .831 & .418 \\
\hline & Duration dysphagia & 25.188 & 109.949 & .046 & .229 & .822 \\
\hline & $\mathrm{BMI}$ & 1.162 & 1.367 & .214 & .850 & .408 \\
\hline
\end{tabular}

a Dependent Vanable: follow up 
During the study period a total of seven patients, (6.5\%), developed complications relevant to follow up survival. One patient developed recurrent dysphagia secondary to confirmed malignant stricture. Two patients developed recurrent dysphagia, which was assumed malignant as they were lost to follow up immediately thereafter unlike their counterparts who developed benign strictures. And four patients developed clinical features of disease spread with appearance of new enlarged cervical nodes, liver nodules, ascites and pulmonary features of malignant secondaries. For two patients in this latter group, the edges of the resected oesophagus displayed invasion with tumour suggesting associated inadequate margin resection.

\section{Discussion}

The two significant independent prognostic factors following oesophagectomy that influence survival are the spread of tumour through the oesophageal wall and the presence of lymph node metastasis $(8,9,10,12,14)$. The presence of metastasis to the regional nodes is a fairly common occurrence. Isono et al in their series found node metastasis present in up to $32 \%$ of neck nodes and about half of the nodes in the chest and abdomen at surgery (11).

Even though not statistically significant, the same nodal trends were observed in our study; with a greater number of enlarged regional nodes having a slightly shorter survival (Figure 3). Follow-up and tumour spread beyond the oesophageal serosal wall confirmed a slightly shortened follow up (Figure 4). Again this was not statistically significant.

The degree of cellular differentiation on histology was found to have no relationship to the follow up pattern. This would have been expected to be the most reliable prognostic indicator to the follow up pattern yet failed to reach significance. As the majority of the histology reports were endoscopic compared to final histological specimens, it could be that the sampling via endoscope was possibly not representative of the true state of differentiation of the full specimen. This may explain why a few endoscopic results reported as carcinoma in situ, which would be expected to be early disease, was actually reported in patients presenting with grade three or four dysphagia.

There were seven patients who developed clinical signs suggestive of recurrences. This brings into focus the need to accurately stage the spread of diseased in the pre-operative period. Looking at the cost benefit to the institution, the savings made investing in investigative equipment will most possibly be more than compensated by the savings in not operating on patients with unresectable disease.

Apart from the above factors, other factors influencing postoperative survival but are not significant independent prognostic factors include patient age, size of the tumour, duration of symptoms, nutritional status and postoperative complications amongst others $(15,16)$.

Looking at our other study variables only low preoperative levels of serum albumin and protein are statistically significant in relation to shortened follow up. Oesophageal carcinoma in common with a lot of other malignancies results in significant weight loos commonly referred to as cancer cachexia. This syndrome results in both physiological as well as metabolic derangements leading to weight loss $(3,17)$. Restoration of normal feeding is not a guarantee that preoperative weight will be restored even using specialised nutritional support programmes. As yet unpublished data for this hospital shows that fifty percent of our oesophagectomy patients continue to loose weight postoperatively. This is probably a reflection of advanced disease and the multiple causes of cancer cachexia, not all of which respond to restored nutritional intake. It is possible this shortened follow up is accounted for by this group of patients with significant cachexia reflected through serum biochemistry.

Overweight patients seemed to have a shorter follow up compared to their normal or underweight counterparts in this study. Overweight patients in the western populations tend to present with a higher proportion of adenocarcinoma at the cardia which in turn is associated with earlier spread through the celiac nodes and shorter postoperative survival (18). Despite being overweight they tend to be 
disadvantaged by having more extensive nodal spread at presentation; and this may account for the shorter follow up amongst this group of patients.

Patients having extended surgical resection show no long term survival advantage over conventional resection or trans-hiatal resection once nodal spread is present (19). A similar follow up trend was demonstrated in the current study. This further suggests the need for acurate pre-operative staging (20-23).

Poor infrastructure has for long been blamed in this country as being one of the reasons for poor follow up of our hospital patients in general. The assumption being the further the distance from the hospital, the more of a financial burden it is to travel to the hospital and the poorer the follow up. This study shows no correlation between the distances from the hospital to the homes of the patients to suggest that distance has any influence on follow up. Patients are either more enlightened and making all efforts to come for follow-up irrespective of locality, or possibly more of our elderly patients have able relatives in Nairobi able to finance clinic follow up compared to twenty or so years back.

The follow up pattern for this study was statistically different from the retrospective analysis earlier published for this hospital covering the period January 1987 to January 2001, $(p=0.047)$, with one, three and five year follow up rates then of $43 \%, 22 \%$ and $10 \%$ respectively. (20). What may be the possible explanation for this discrepancy? Over the last ten or so years the availability of surgical services in the district hospitals has increased tremendously in this country and more than $50 \%$ now have a surgeon on site. As a result, a lot of surgical procedures and facilities for follow up previously not available in the rural communities are now available closer to them. Subsequently many of our patients are opting for follow up in their rural facilities rather than coming back to the $\mathrm{KNH}$; and indeed do make such requests to us. This development is likely to be the reason our prospective follow up analysis is lower than our previous retrospective follow up pattern.

Surgical clinic follow up and post operative survival are not necessarily the same and this study would like to acknowledge this. However the study does also appreciate the two, though different, have similar trends and until an effective mode of communication with patients post discharge can be put in place, follow up will continue to be our main variable for analysis. The study also appreciated the use of an end point other than three months will have an influence on the follow up pattern. This figure was used as this tended to be the average period given to our patients for their appointments.

\section{Acknowledgments}

Appreciation is expressed to the office of the Director of the Kenyatta National Hospital for permission to carry out this study and to publish the data. Also to all the doctors and nurses who may in any way have assisted in the data collection.

\section{References}

1. Abo S., Kitamura M., Hashiinoto M., et al. Analysis of results of surgery performed over a twenty year period on 500 patients with cancer of the thoracic oesophagus. Jpn J. Surg. 1996; 26: 77-82.

2. Shao L.F., Gao Z.G., Yang N.P., et al. Results of surgical treatment of 6123 cases of carcinoma of the oesophagus and gastric cardia. J. Surg. Oncol. 1989; 42: 170-174.

3. Kern K.A. and Norton J.A. Cancer cachexia. J. Parent Ent. Nutr. 1988; 12: 286-298.

4. Shao L.F., Gao Z.G., Yang N.P., et al. Results of surgical treatment of 6123 cases of carcinoma of the oesophagus and gastric cardia. J. Surg. Oncol. 1989; 42: 170-174.

5. King R.M., Pairolero P.C., Trastek V.F., et al. Ivor-Lewis oesophagectoiny for carcinoma of the oesophagus: Early and late functional results. Ann. Thorac. Surg. 1987; 44: 199-122.

6. Matsubara J., Kaise T., Ishiguro M. and Nakajima $\mathrm{T}$. Better grading systems for evaluating the degree of lymph node invasion in cancer of the thoracic oesophagus. Jpn. J. Surg. 1994; 24: 500-505.

7. DeVita V.T., Hellinan S. and Rosenburg S.A. Cancer: Principles and practice of oncology. J.B. Lippincoll Company 1993, 4th edition. 776-817. 
8. Teruo Kakegawa. Forty years experience in surgical treatment of oesophageal cancer. Int. J. Clin. Oncol. 2003; 8: 277-288.

9. Skinner D.B., Little A.G., Ferguson M.K., et al. Selection of operation for oesophageal cancer based on staging. Ann. Surg. 1986; 204: 391-401.

10. Helm Z., Yumin P. and Xiquri D. Study on factors influencing survival in patients with cancer of the oesophagus after resection by Cox proportional hazard model. Chinese J. Oncol. 1999; 21: 32-34.

11. Isono K., Ochiai T., Okuyama K. and Onoda S. The treatment of lymph node metastasis from esophageal cancer by extensive lymphadenectoiny. Jpn J. Surg. 1990; 20: 151-157.

12. Tachibana M., Dhar D.K., KinugasaS., et al. Esophageal cancer patients surviving 6 years after esophagectoiny. Langenbeck's Arch. Surg. 2002; 387: 77-83.

13. Nozoe T. and Sugimachi K. Simple staging criteria for oesophageal carcinoma classification with a strict prognostic stratification. Amer. J. Clin. Oncol. 2003; 26: 307-331.

14. Baba M., Natsugoe S., Kusana C., et al. Lymph node metastasis and the recurrence of oesophageal carcinoma with emphasis on lymphadenectoiny in the neck and superior inediasternuin. Jpn. J. Surg. 1995; 25: 125-130.

15. Hirai T., Yainashita Y., Mukaida H., etal. Poor prognosis in oesophageal cancer patients with postoperative complications. Jpn. J. Surg. 1998; 28: 576-579.
16. Tsutsui S., Sonoda K., Suiniyoshi K., et al. Prognostic significance of immunological parameters in patients with oesophageal cancer. Hepato-Gastroenterol. 1996; 43: 501-509.

17. Brennan M.F. Total parenteral nutrition in the cancer patient. New Engl. J. Med. 1981; 305: 375-382.

18. Alexiou C., Khan O.A., Black E., et al. Survival after oesophageal resection for carcinoma: The Importance of the Histologic Cell Type. Ann. Thorac. Surg. 2006; 82: 1073-1077.

19. Bolton J.S. and Teng S. Transthoracic or transhiatal esophagectomy for cancer of the esophagus - Does it matter? Surg. Oncol. Clin. North Amer. 2002; 11: 365-375.

20. Ogendo S.W.O. Follow up of oesophageal cancer therapy at the Kenyatta National Hospital, Nairobi. East Afr. Med. J. 2001; 78: 650-654.

21. Ali A., Ersuino T. and Johnson O. Oesophageal carcinoma in Tikur Anbessa Hospital, Addis Ababa. East Afr. Med. J. 1998; 75: 590-593.

22. Abmed M.E. The surgical management and outcome of oesophageal cancer in Khartoum. JR Coll. Surg. Edinb. 1993; 38: 16-8.

23. Sumeruk R., Segal I., Te Winkel W. and Van der Merwe C.F. Oesophageal cancer in three regions of South Africa. South Afr. Med. J. 1992; 81: 91-93. 